\title{
Revisión bibliográfica de la capacidad funcional en trabajadores mayores de 65 años
}

\section{Valuation of the functional capacity of the 65 years old major workers}

\author{
Beatriz de León Luis ${ }^{1,3}$, Schyrley Díaz ${ }^{2,3}$ \\ 1. Mutua Accidentes de Canarias \\ 2. Unidad Docente. Hospital Universitario Doctor Negrín. Gran Canaria \\ 3. Escuela Nacional de Medicina del Trabajo. Instituto de Salud Carlos III. Madrid. España. \\ Recibido: 30-01-11 \\ Aceptado: 21-02-11
}

\section{Correspondencia}

Beatriz de León Luis

Bencomia, 2 (Urbanización Mayber)

38296 San Miguel de Geneto. LA LAGUNA. (Tenerife). España.

Tfnos: 922260780; 922256946;648228523

Email: lelubea@gmail.com; Lelubea@hotmail.com; bleon@cusmet-enmt.isciii.es

\section{Resumen}

Introducción: Los países industrializados están sufriendo importantes transformaciones en su evolución demográfica, caracterizado por el envejecimiento de la población (disminución de la tasa de natalidad, incremento de la población adulta y aumento de la esperanza de vida). En España el decremento de la tasa pensionista/ cotizante conlleva a que en la actualidad se esté discutiendo en el gobierno el retraso en la edad de jubilación.

Justificación: En el futuro próximo posiblemente los empleadores se enfrentarán con el dilema de la planificación de las tareas en trabajadores sensibles, deberán diseñar herramientas o modificar condiciones de trabajo para garantizar la salud y capacidad de trabajo de sus trabajadores mayores. Sin embargo antes de cualquier intervención, los entes responsables deben conocer las necesidades exactas de esta población laboral.

Objetivo: Esta revisión pretende considerar los cambios fisio-patológicos del envejecimiento y documentar la bibliografía que abarca las acciones a seguir por parte del médico de trabajo para una correcta valoración integral de la capacidad funcional, en consideración o no de las distintas profesiones.

Metodología: Se utilizaron las bases de datos bibliográficas MEDLINE (PUBMED) y OSH UPDATE. Se revisaron a texto completo aquellos artículos dirigidos a la valoración de la capacidad funcional y efecto del envejecimiento en la capacidad del trabajo. Resultados y conclusiones: La evaluación de la capacidad funcional de los trabajadores mayores debe incluir: evaluación de la capacidad física, mental, social; evaluación de cualquier discapacidad; así como percepción individual de su capacidad de trabajo: WAI (Work capacity index).

Med Segur Trab (Internet) 2011; 57 (222) 63-76

Palabras claves: Adulto mayor, Envejecimiento del trabajador, Evaluación de la capacidad del trabajo, Capacidad funcional, Sentidos.

Abstract

Introduction: Industrialized countries are suffering important transformations in their demographic evolution, characterized by the aging of the population (decrease in the birthrate, increase in adult population 
and increased life expectancy). In Spain the decrease in the rate pensioner/contributor means the government is discussing the possibility of a delay in the retirement age.

Justification: In the near future employers will probably face the dilemma of planning tasks for sensitive workers, designing tools or modifying conditions of work to guarantee the health and work capacity of elder workers. However before any intervention, the responsible entities must know the exact needs of this working population.

Objective: This review tries to consider the fisio-pathological changes of the aging population and to document the bibliography that includes the actions to be taken by the labor physician for a correct integral assessment of the functional capacity, bearing in mind or not the different professions.

Methodology: Bibliographical databases such as MEDLINE (PUBMED) and OSH UPDATE were used. Complete revision was made of those articles about the assessment of the functional capacity and the effect of aging in the work capacity.

Results and conclusions: The assessment of the functional capacity of elder workers must include: assessment of physical, mental and social capacity; assessment of any disability, as well as individual perception of his capacity for work: WAI (Work capacity index).

Med Segur Trab (Internet) 2011; 57 (222) 63-76

Key words: Elder worker, Aging worker, work capacity evaluation, functional capacity, senses. 


\section{INTRODUCCIÓN}

La evolución demográfica de los países industrializados está sufriendo importantes transformaciones $\mathbf{1 , 2}^{\mathbf{2}}$. La pirámide de edad de edad poblacional tiende a abandonar su forma clásica para asemejarse a la de pera invertida, tal como se evidencia en la siguiente figura:

Figura 1. Fuente: Perspectivas de población en el mundo. Revisión de 2008, ONU.

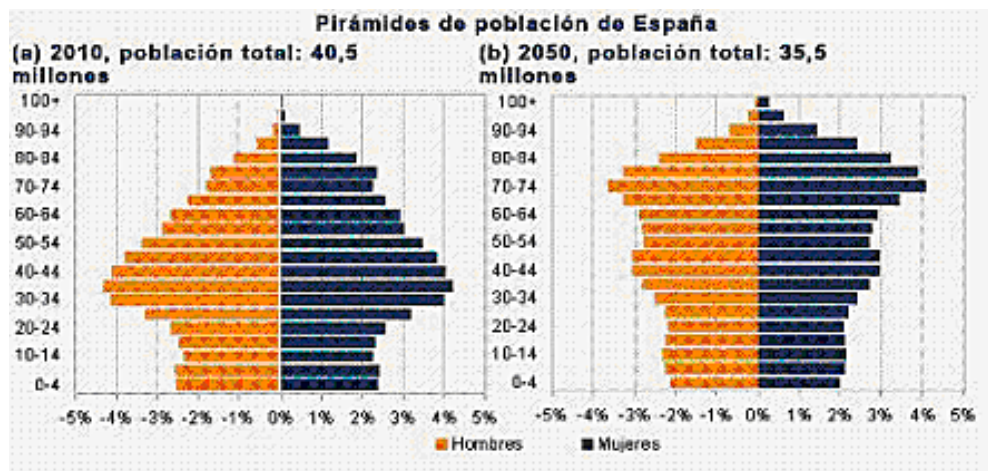

La causa principal de esta inversión es el descenso de las tasas de natalidad (figura 2),

Figura 2. Tasa de natalidad española, Indicadores Demográficos Básicos, 2009. Indicador Coyuntural de la Fecundidad (número medio de hijos por mujer

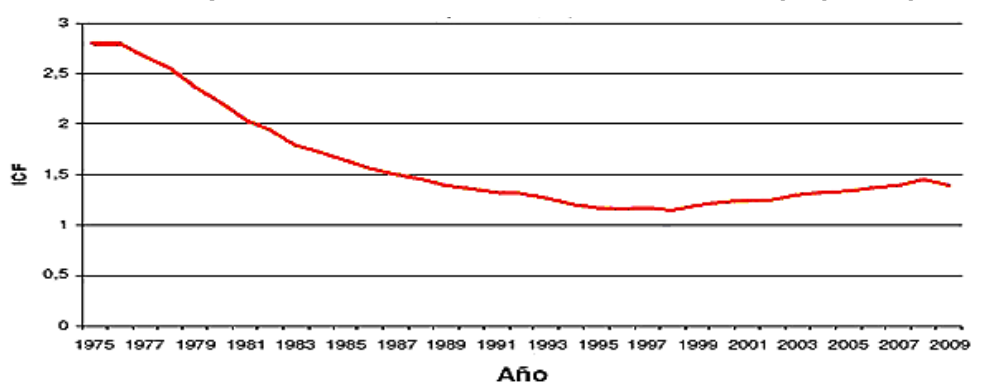

asociado al incremento de la población de edad adulta y el incremento de la esperanza de vida (figura 3), relacionado con las mejoras en las condiciones de salud y de vida ${ }^{1,2}$.

Figura 3. Esperanza de Vida, Indicadores Demográficos Básicos, 2009

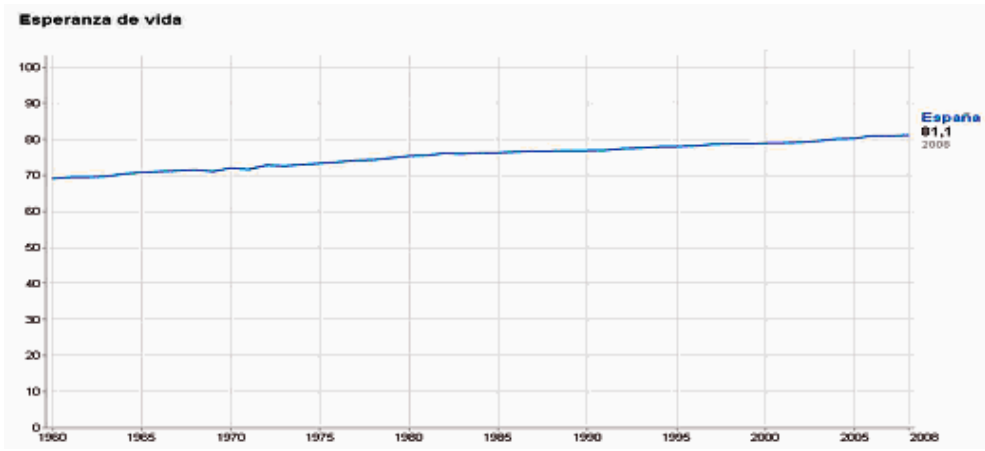

La OECID (Organización para el Desarrollo y Cooperación Económica) ${ }^{2}$ estima que en sus 24 países miembros (entre los que se incluye España), la proporción de trabajadores con edades entre 45 y 64 años respecto a la población total de trabajadores (de 14 a 64 años) pasará del 32\% de 1980 al 41,3\% esperado para el 2025; es decir, para esta fecha algo menos de la mitad de los trabajadores tendrá más de 45 años ${ }^{2,3}$. Dos consecuencias importantes que cabe esperar de esta situación y que justifican la consideración del envejecimiento y su relación con el trabajo como objeto de estudio son en primer lugar la gran proporción de mano de obra constituida por trabajadores de edades avanzadas 
(más de 50 años) y el decremento de la tasa pensionista-cotizante (en España se estima que, si se mantienen las tendencias apuntadas, habrá menos de dos cotizantes por pensionista en el año 2020) lo que implica la imposibilidad de que la fuerza productiva mantenga a los pensionistas en los términos actuales. Cabe esperar de esto que la vida laboral se alargue, retrasándose la edad de jubilación. ${ }^{2,4}$

El crecimiento del sector de más edad de la población plantea dudas sobre la capacidad de los estados para cumplir con sus obligaciones financieras. Más concretamente, sobre su capacidad para generar recursos que garanticen las pensiones y cuidados requeridos por las personas mayores, especialmente en una situación de crisis económica como la actual: Siendo menos los contribuyentes ¿cómo pueden proporcionar recursos para un número crecientes de personas jubiladas? Para poder proporcionar respuesta satisfactoria a la anterior pregunta, cada vez son más lo que abogan por un retraso en la edad de la jubilación ${ }^{5,6}$. España no escapa de esta situación, por ello en la actualidad el gobierno ha planteado al Consejo de Ministros propuesta para modificar la ley y retrasar dos años la edad de jubilación en España. Sin embargo, pese a las necesidades económicas, dos preguntas claves que plantemos en el marco de la medicina del trabajo son: ¿Si están los trabajadores mayores de 65 años preparados para trabajar dos años más?, ¿Existen revisiones bibliográficas que informen al médico de trabajo cómo hacer una valoración física y funcional al trabajador de edad avanzada para aprobar o dar continuidad a una aptitud laboral?

La capacidad física del trabajador ocupa un lugar destacado en el siguiente modelo:

Figura 4. Modelo de envejecimiento y carga física de Shephard RJ, 1999

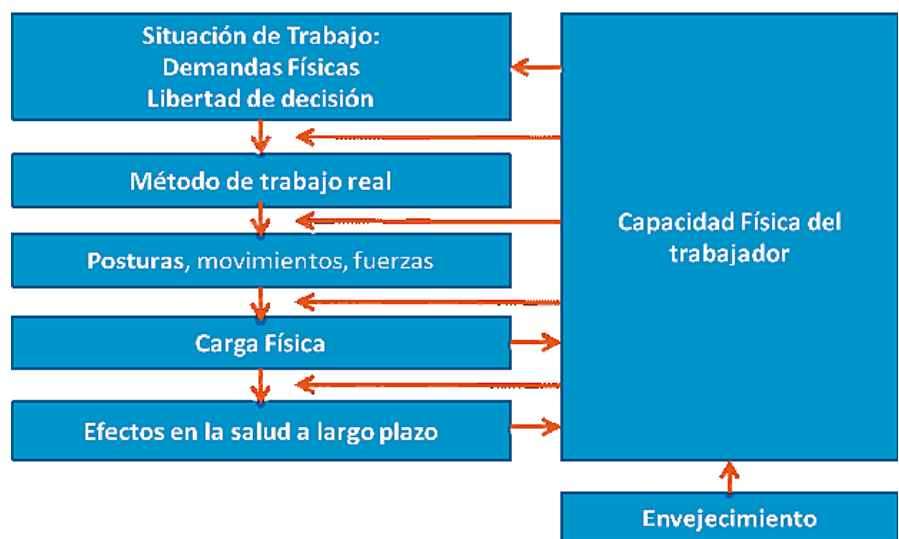

La cual se ve afectada por tres factores determinantes: la carga física, efectos en la salud a largo plazo y el propio envejecimiento ${ }^{7,8}$.

Antes de detallar los aspectos relacionados con la valoración de la capacidad funcional, es importante resaltar de forma sencilla cuáles son los cambios fisiopatológicos del envejecimiento que debemos considerar:

A nivel Respiratorio el proceso del envejecimiento produce una disminución de los flujos y volúmenes a partir de los 30 años, mientras que a nivel cardiovascular ${ }^{9}$ hay presencia de arteriosclerosis y aumento de resistencias periféricas, con alteraciones cardioeléctricas por degeneración del tejido de conducción (disminuyen las células del automatismo) y se presentan alteraciones del ritmo. En la esfera músculo-esquelético es frecuente encontrar pérdida de masa ósea, osteoporosis, pérdida de masa muscular, degeneración del cartílago articular y Artrosis ${ }^{10}$. A nivel renal el filtrado glomerular se encuentra disminuido. Neurológicamente hay cambios cerebrales, arterioesclerosis, atrofia cerebral, disminución de neurotransmisores, enlentecimiento de los reflejos, menor tolerancia al dolor, marcha senil, disminución de la memoria y del aprendizaje. Tardan más en iniciar acción motora (tiempos de reacción altos) y tienen más dificultades para realizarla ${ }^{11}$. 
Entre las capacidades sensoriales que experimentan un deterioro importante cabe destacar la visión y la audición. La necesidad de atender prioritariamente a estos dos sentidos viene dada tanto por la magnitud de su deterioro como por la importancia que tienen en el proceso de relación de las personas con el entorno exterior y principalmente en el desarrollo de sus tareas dentro de la esfera laboral ${ }^{11}$. La visión se ve deteriorada por el proceso normal de envejecimiento del ojo, pudiendo resultar estos problemas de visión agravados por la existencia de alguna enfermedad degenerativa en el ojo, situación que con la edad tiene mayor probabilidad de ocurrencia. ${ }^{2,12}$

El proceso de envejecimiento altera las propiedades ópticas del globo ocular (pérdida de transparencia y amarillamiento), reduce la nitidez de las imágenes retiniana y altera su cromatismo. Además reduce la eficacia de los mecanismos nerviosos que compensan las variaciones en los niveles de iluminación (cuesta más adaptarse a los cambios en el nivel de iluminación y es más fácil sufrir deslumbramientos). También reduce la capacidad para combinar la información de los dos ojos para recibir la profundidad y por tanto reduce la coordinación ojo-mano y se produce una reducción del campo visual ${ }^{12}$. En relación a la audición, el envejecimiento deteriora la capacidad auditiva especialmente relacionada con las frecuencias altas (mayores a $1.000 \mathrm{~Hz}$ ). El proceso de deterioro auditivo por audiometría se detecta a partir de la tercera década de vida, sin embargo sólo producen efecto funcionalmente importante a partir de la sexta década de la vida. Las pérdidas auditivas tienden a afectar más a los hombres que a las mujeres. Las pérdidas en la capacidad auditiva características de las personas mayores es la principal causa de sus dificultades para entender el lenguaje hablado. ${ }^{12,13}$

\section{OBJETIVO}

Disponer un conocimiento sobre la evidencia de documentación existente en relación a la valoración de la capacidad funcional de los trabajadores mayores de 65 años de y los instrumentos útiles en la consulta para garantizar el estado de aptitud del trabajador.

\section{METODOLOGÍA}

\section{Búsqueda}

Esta revisión centro como únicas fuentes de información las bases de datos bibliográficas MEDLINE (PUBMED) por ser la principal base de datos en National Library of Medicine y OSH UPDATE, por su mayor enfoque en temas relacionados con Salud Laboral y Medio ambiente.

Esta estrategia de búsqueda se ha desarrollado combinando los siguientes descriptores:

Older, Workers, Aging Worker, Work Capacity Evaluation, Capacity Functional, Elderly Workers, Senses, Visual Acuity, Aptitude Test, Vision Test, Health, Occupational

Se hizo la búsqueda de artículos en base a referencias bibliográficas señaladas en dos publicaciones seleccionadas. Se realizaron búsquedas de datos o actualizaciones en páginas web de Organismos Nacionales e Internacionales (Estadísticas y guía de Valoración geriátrica de Atención Primaria).

La última fecha de búsqueda fue el 19 de enero de 2011.

La Tabla 1 que se presenta a continuación reporta un listado de los diferentes términos de búsqueda empleados en las dos bases de datos, así como el número de artículos obtenidos: 
Tabla 1. Estrategia de Búsqueda.

\begin{tabular}{|c|c|c|c|}
\hline Base de datos & Descriptores & Límites & N..$^{\circ}$ Articulos \\
\hline \multirow{18}{*}{ PUBMED } & Work ability index in worker & Sin límites & 11 \\
\hline & Older worker & Sin límites & 0 \\
\hline & Occupational health in worker elderly & Medline, +65 años, ultimos 3 & 55 \\
\hline & Occupational health in worker older & Ultimos 10 años, +65 años & 22 \\
\hline & Older workers and senses & Medline, +65 años & 1 \\
\hline & Evaluation changes in aging worker & Sin límites & 7 \\
\hline & Cognitive assessment in aging worker & Sin límites & 1 \\
\hline & Evaluation hearing on aging worker & Sin límites & 0 \\
\hline & Visual assessment in older worker & Sin límites & 0 \\
\hline & Sensory impairment in older workers & Sin límites & 1 \\
\hline & Older worker and ergonomic & Medline, +65 años & 5 \\
\hline & Work capacity evaluation & Últimos 5 años, +65 años & 12 \\
\hline & Older worker and senses & Mayores a 65 años & 1 \\
\hline & Work capacity evaluation and aging worker & Mayores a 65 años & 3 \\
\hline & Elderly workers and capacity functional & Idiomas, mayores a 65 años & 30 \\
\hline & Vision test and aging worker & Mayores a 65 años & 0 \\
\hline & Visual acuity and workers & Idiomas, mayores a 65 años & 20 \\
\hline & Aptitude test and workers & Mayores a 65 años & 5 \\
\hline \multirow{11}{*}{$\begin{array}{c}\text { OSH } \\
\text { UPDATE }\end{array}$} & Work ability index & Sin límites & 32 \\
\hline & Work ability index in worker & Sin límites & 0 \\
\hline & Occupational health in worker elderlys & In límites & 0 \\
\hline & Older workers and senses & In límites & 2 \\
\hline & Evaluation changes in aging worker & Sin límites & 0 \\
\hline & Cognitive assessment in aging worker & Sin límites & 0 \\
\hline & Hearing assessment in older workers & Sin límites & 0 \\
\hline & Visual assessment in older worker & Sin límites & 0 \\
\hline & Sensory impairment in older workers & Sin límites & 0 \\
\hline & Older worker and ergonomic & Sin límites & 8 \\
\hline & Work capacity evaluation & Sin límites & 5 \\
\hline
\end{tabular}

\section{Selección de Artículos}

Se revisaron los resúmenes de los artículos encontrados con la estrategia de búsqueda y se seleccionaron aquellos que cumplían los criterios de inclusión, descartando a su vez según los criterios de exclusión establecidos para esta revisión.

Criterios de inclusión:

1. Publicaciones dirigidas a la valoración de la capacidad funcional y efecto del envejecimiento en la capacidad del trabajo.

2. Estudios publicados en inglés, español o italiano.

3. Estudios internacionales o nacionales.

Criterios de exclusión:

1. Publicaciones anteriores a 1994.

2. Artículos que no relacionados con el envejecimiento.

3. Valoraciones de patologías concretas (morbilidad asociada) en trabajadores mayores.

La principal limitación encontrada en la realización de la revisión corresponde a numerosas publicaciones relacionadas con patologías concretas (comorbilidad) muy frecuente en los adultos mayores (ejemplo: cánceres, cardiopatías, depresión...) desvirtuando nuestro objetivo de sistematizar publicaciones que enfoquen la valoración 
del trabajador mayor de 65 años como un individuo sano y/o competente capaz de continuar con sus actividades laborales como el resto de los trabajadores.

\section{RESULTADOS}

Se obtuvieron un total de 221 artículos con la búsqueda y tras aplicar los criterios de inclusión y exclusión mencionados anteriormente se seleccionaron un total de 21 publicaciones a texto completo, de los cuales se presentaran a continuación conceptos generales importantes que enfocan la valoración.

El término capacidad funcional describe la capacidad de un individuo para realizar su trabajo con respecto a las demandas laborales específicas ${ }^{14}$. Aunque las demandas de trabajo a menudo sean las mismas, los trabajadores experimentan cambios en las capacidades físicas y mentales a medida que envejecen. La evaluación debe consistir en el análisis de las tareas y la cuantificación de las exigencias físicas del trabajo, observación de parámetros fisiológicos y psicológicos, las demandas psicosociales y los problemas de salud asociados ${ }^{6}$.

Un adulto mayor suele presentar comorbilidad asociada, la mayoría de estas patologías corresponden a enfermedades crónicas que suelen estar controladas, sin embargo el médico debe considerarlas y medir su impacto individual en el individuo activo mayor de 65 años, tales escalas son: índice de Charlson, índice de enfermedad coexistente y el índice de Kaplan?.

Según Parker la valoración va enfocada de la siguiente forma: patología o discapacidad presentada, tipo de trabajo (tareas), rehabilitación y capacidad de retornar al trabajo. Se debe evaluar la productividad y medir los factores de riesgo propios de la edad ${ }^{15}$. Deben existir medidas ergonómicas por sus cambios fisiopatológicos y no solo se debe ajustar al trabajador mayor al lugar de trabajo sino conseguir trabajos adecuados para ellos ${ }^{3}$.

Los riesgos propios de la edad a considerar en la valoración de un adulto mayor de 65 años son: cumplimiento del esquema de vacunación (antigripal-antitetánica), tabaquismo, alcoholismo, hábitos higiénicos, hábitos dietéticos, actividad física, Hipertensión arterial, dislipidemias, diabetes, Osteoporosis, Índice de masa corporal, Riesgo Cardiovascular, Cáncer de endometrio, Cáncer de mama, Trastornos sensoriales (Visión, audición), exploración buco-dental, riesgos de polimedicación, riesgo de caídas, despistaje de incontinencia urinaria ${ }^{9,11}$.

Los resultados de las publicaciones revisadas se resumen en 5 tablas que se presentarán a continuación, las cuales fueron divididas según los parámetros de valoración:

Tabla 2. Valoración de la capacidad física.

\begin{tabular}{|c|c|c|c|c|c|}
\hline $1^{\circ}$ Autor & Año & Artículo & Tipo de estudio & Población & Capacidad física \\
\hline \multirow[t]{2}{*}{ Popkin S } & \multirow[t]{2}{*}{2008} & \multirow{2}{*}{$\begin{array}{l}\text { La edad es algo más } \\
\text { que un número }\end{array}$} & \multirow{2}{*}{$\begin{array}{l}\text { Revisión } \\
\text { sistemática }\end{array}$} & \multirow{2}{*}{$\begin{array}{c}\text { Trabajadores } \\
\text { del sector de } \\
\text { transporte }\end{array}$} & Agudeza visual \\
\hline & & & & & $\begin{array}{c}\text { Riesgo de } \\
\text { muerte súbita }\end{array}$ \\
\hline $\begin{array}{l}\text { Servicio } \\
\text { Canario } \\
\text { de Salud }\end{array}$ & 2002 & $\begin{array}{l}\text { Guía de Atención en } \\
\text { las personas Mayores } \\
\text { en Atención Primaria }\end{array}$ & Texto completo & & $\begin{array}{c}\text { Valoración } \\
\text { Integral }\end{array}$ \\
\hline \multirow[t]{2}{*}{ Sluiter J. } & \multirow[t]{2}{*}{2006} & \multirow{2}{*}{$\begin{array}{l}\text { Alta demanda de } \\
\text { puestos de trabajo: } \\
\text { la diversidad }\end{array}$} & \multirow[t]{2}{*}{$\begin{array}{l}\text { Revisión } \\
\text { sistemática }\end{array}$} & & $\begin{array}{c}\text { Profesiones de } \\
\text { alta demanda }\end{array}$ \\
\hline & & & & & $\begin{array}{c}\text { Uso de pruebas } \\
\text { especiales }\end{array}$ \\
\hline Persad C & 2010 & $\begin{array}{l}\text { Evaluación de las caídas } \\
\text { en los ancianos }\end{array}$ & $\begin{array}{c}\text { Revisión } \\
\text { sistemática }\end{array}$ & & $\begin{array}{l}\text { Riesgo de } \\
\text { caídas }\end{array}$ \\
\hline
\end{tabular}




\begin{tabular}{|c|c|c|c|c|c|}
\hline $1^{\circ}$ Autor & Año & Artículo & Tipo de estudio & Población & Capacidad física \\
\hline \multirow[t]{2}{*}{ Ilmarinem $\mathrm{J}$. } & \multirow[t]{2}{*}{2000} & \multirow{2}{*}{$\begin{array}{l}\text { Envejecimiento de la } \\
\text { población trabajadora } \\
\text { de la UE }\end{array}$} & \multirow[t]{2}{*}{$\begin{array}{l}\text { Revisión } \\
\text { sistemática }\end{array}$} & & $\begin{array}{l}\text { Carga de trabajo/ } \\
\text { condiciones físicas }\end{array}$ \\
\hline & & & & & $\begin{array}{c}\text { Fisiopatología } \\
\text { del envejecimiento }\end{array}$ \\
\hline Kenny G. & 2008 & $\begin{array}{l}\text { Capacidad Física en } \\
\text { trabajadores mayores: } \\
\text { implicaciones del } \\
\text { envejecimiento }\end{array}$ & $\begin{array}{l}\text { Revisión } \\
\text { sistemática }\end{array}$ & & $\begin{array}{c}\text { Accidentes de } \\
\text { trabajo/Ejercicio }\end{array}$ \\
\hline $\begin{array}{l}\text { Kowalski- } \\
\text { Trakofler K. }\end{array}$ & 2005 & $\begin{array}{l}\text { Condiciones de } \\
\text { seguridad para el } \\
\text { envejecimiento } \\
\text { en la fuerza laboral }\end{array}$ & $\begin{array}{l}\text { Revisión } \\
\text { sistemática }\end{array}$ & & $\begin{array}{c}\text { Valoración } \\
\text { musculo-esquelética / } \\
\text { condiciones extremas }\end{array}$ \\
\hline Chang G & 2000 & $\begin{array}{l}\text { Envejecimiento y } \\
\text { aptitud } \\
\text { para trabajar }\end{array}$ & $\begin{array}{l}\text { Revisión } \\
\text { sistemática }\end{array}$ & & $\begin{array}{l}\text { Agudeza visual, } \\
\text { auditiva y músculo- } \\
\text { esqueléticas }\end{array}$ \\
\hline Dávila E. & 2009 & $\begin{array}{l}\text { Deterioro sensorial } \\
\text { entre } \\
\text { los trabajadores } \\
\text { mayores EE.UU }\end{array}$ & $\begin{array}{l}\text { Descriptivo: } \\
\text { Corte de } \\
\text { registros } \\
\text { históricos }\end{array}$ & $\begin{array}{l}\text { Casi } 4 \text { millones } \\
\text { de trabajadores } \\
>65 \text { años, ENS } \\
\text { de EUA (1997- }\end{array}$ & $\begin{array}{c}\text { Déficit } \\
\text { sensitivo }\end{array}$ \\
\hline
\end{tabular}

en la Capacidad Física: 4 relacionados con capacidad musculo-esqueléticas, 6 de ellos relacionados con la valoración sensorial y dos de ellos hacen referencia a restricciones.

La evaluación médica debe incluir anamnesis detallada y dirigida a las tareas, sus déficits y capacidades potenciales, exploración física completa, detección de factores de riesgo y cumplimiento de actividades preventivas ${ }^{11}$.

Los cambios del envejecimiento físico incluyen el deterioro físico, sensitivo y procesos motores que pueden limitar la capacidad funcional durante el proceso de envejecimiento. La audición, visión, fuerza, balance, movilidad articular, destreza manual, tiempos de reacción y movimiento y resistencia, todas sufren deterioro con la edad, y algunos trabajadores sufren de enfermedades degenerativas articulares, osteoporosis, enfermedad cardiovascular y desórdenes metabólicos ${ }^{16}$.

Los cambios musculo-esqueléticos más significativos relacionados con la edad ocurre en las articulaciones, especialmente para realizar pequeños movimientos como agarre, giros y movimientos grandes como caminar, flexionar, sentarse, escalar, parar y reiniciar la marcha, A pesar de que la movilidad articular disminuye lentamente desde los 20 a los 60 años y la incidencia de artritis aumenta dramáticamente a partir de los 45 años. En relación a la fatiga de los músculos del hombro en trabajadores mayores que experimentan disminución de la movilidad articular, han sido asociados con trabajos que requieren la elevación de los brazos. A los trabajadores que manipulan cargas deben realizarse especial valoración musculo-esquelética ${ }^{17}$. La pérdida del músculo esquelético termina en una disminución de la masa corporal. La fuerza muscular disminuye con la edad a una tasa más rápida cerca de los 50 años ${ }^{16}$.

El riesgo de caída por alteraciones sensoriales y musculo esqueléticas aumenta en la población mayor. Es importante la implementación de una medida de evaluación de riesgo de caídas (desde cuestionarios simples hasta evaluaciones neurológicas y vestibulares) ${ }^{18}$. El 25\% de los trabajadores entre 55-64 años están obligados a disminuir carga de trabajo por condiciones físicas ${ }^{14}$. Una mayor edad está asociada con una alta prevalencia de deterioro sensitivo que a su vez es asociado con riesgo aumentado de accidentes de trabajo. En los trabajadores mayores de 65 años de EUA, la prevalencia de la discapacidad auditiva es 3 veces mayor que la discapacidad visual (33.3\% vs $10.2 \%)$. En relación a las ocupaciones, los operadores de granja, pesca, construcción y los gerentes reportaron el mayor deterioro visual (15.4\%), deterioro auditivo (53.9\%), ambos deterioros (12.1\%) y algún deterioro (57.3\%). Hay 2 posibles explicaciones para estos hallazgos: 
primero, el deterioro auditivo podría ser causado por la exposición ocupacional dañina como altos niveles de ruido, los cuales están bien documentados entre granjeros, trabajadores de la construcción y operarios de maquinaria. El deterioro visual podría ser causado por incrementos en los factores de riesgo de enfermedad ocular (p.e exposición solar) y lesiones oculares (p.e exposición a químicos, polvo, radiación, soldaduras, productos agrícolas, penetración de cuerpos extraños) los cuales son frecuentes en los trabajadores de custodia, reparadores de casas, salud, agricultura e industrias manufactureras ${ }^{19}$.

Popkins ${ }^{18}$, encontró evidencia de que la agudeza visual y la sensibilidad al contraste disminuían con la edad, por lo que la exploración de agudeza visual y búsqueda de enfermedades oculares en este grupo etario debe ser rutinario. Reporta en su artículo que los pilotos deben retirarse de vuelos comerciales a partir de los 60 años por el riesgo elevado de muerte súbita o accidente cardiovascular durante el vuelo. Los controladores aéreos deben retirarse a los 61 años, por riesgo de agotamiento físico y mental debido a condiciones laborales exigentes. La exploración de la capacidad Auditiva sugiere realizarla sólo en aquellos puesto con altas demandas o relacionados con el riesgo y la exploración musculo-esquelético, respiratoria, cardiovascular y neurológica cuando haya riesgo de caídas ${ }^{16}$.

Los receptores de presión de localización profunda también parecen disminuir con la edad. Este deterioro puede disminuir la capacidad para detectar señales de presión, incluso de sencillas tareas como presionar un botón para encender una luz. Además, nuestro cuerpo puede ser menos sensible al dolor causado por terminaciones térmicas requiriendo blindaje para calor o frio accidental o prolongado y generando restricciones laborales en este grupo etario, ante condiciones climáticas extremas ${ }^{17}$.

Trabajos de bomberos, trabajadores de ambulancias, agentes de policía, especialistas médicos, pilotos, astronautas y oficiales de submarinos son profesiones con altas demandas físicas que pudiera afectar considerablemente la salud y rendimiento de los trabajadores mayores ya que requieren muchas veces cambios de temperaturas extremas, uso de fuerza física. Requieren una valoración física profunda incluso con ensayos o simulacros de sus condiciones laborales para el mantenimiento de su aptitud, así como pruebas especiales de capacidad funcional cardiovascular y respiratoria ${ }^{6}$. Mientras que Kowalski-Trakofler ${ }^{17}$, señalan que la capacidad aeróbica a los 65 años es cerca del $70 \%$ de la que corresponde a los 25 años. Por lo tanto, ambientes con condiciones climáticas extremas son más exigentes para trabajadores mayores y también recomiendan su restricción ${ }^{17}$.

La capacidad física en trabajadores entre 40-60 años está reducida al menos un 20\% por los procesos fisiopatológicos del envejecimiento. Esta situación pudiera incrementar lesiones relacionadas con el trabajo. Kenny defiende la necesidad de interrogar actividad física habitual como factor preventivo del deterioro y asegura que de continuar con hábitos de vida saludable se puede llegar a la edad de jubilación sin inconvenientes ${ }^{17}$.

\section{Valoración de la capacidad funcional}

De las fuentes consultadas la guía de Atención Primaria ${ }^{9}$ parece ser el más concreto en valorar en esta población el grado de independencia para la realización de las actividades de la vida diaria (AVD), utilizando: Índice de Katz y Barthel para la AVD Básicas y la Escala de Lawton y Brody para las AVD Instrumentales. Estas escalas al medir capacidad para realizar actividades simples rutinarias pierden validez en la esfera laboral, pues se requiere de condiciones físicas y mentales adecuadas sin incapacidad limitante o dependientes de cuidador para asistir a trabajar. 
Tabla 3. Valoración de la capacidad mental.

\begin{tabular}{|c|c|c|c|c|c|}
\hline $1^{\circ}$ Autor & Año & Artículo & Tipo de estudio & Población & Capacidad física \\
\hline $\begin{array}{l}\text { Servicio } \\
\text { Canario } \\
\text { de Salud }\end{array}$ & 2002 & $\begin{array}{l}\text { Guía de Atención en } \\
\text { las personas Mayores } \\
\text { en Atención Primaria }\end{array}$ & Texto completo & & $\begin{array}{l}\text { Valoración } \\
\text { Integral }\end{array}$ \\
\hline Chang G & 2000 & $\begin{array}{l}\text { Envejecimiento y } \\
\text { aptitud para trabajar }\end{array}$ & $\begin{array}{l}\text { Revisión } \\
\text { sistemática }\end{array}$ & & $\begin{array}{l}\text { Memoria y } \\
\text { aprendizaje }\end{array}$ \\
\hline
\end{tabular}

\begin{tabular}{|c|c|c|c|c|c|}
\hline Sluiter J. & 2006 & $\begin{array}{l}\text { Alta demanda de } \\
\text { puestos de trabajo: } \\
\text { la diversidad asociada } \\
\text { a la edad }\end{array}$ & $\begin{array}{l}\text { Revisión } \\
\text { sistemática }\end{array}$ & & $\begin{array}{l}\text { Exlusión de } \\
\text { profesiones }\end{array}$ \\
\hline $\begin{array}{l}\text { Kowalski- } \\
\text { Trakofler K. }\end{array}$ & 2005 & $\begin{array}{l}\text { Condiciones de } \\
\text { seguridad para el } \\
\text { envejecimiento } \\
\text { en la fuerza laboral }\end{array}$ & $\begin{array}{l}\text { Revisión } \\
\text { sistemática }\end{array}$ & & Aprendizaje \\
\hline Popkin S. & 2008 & $\begin{array}{l}\text { La edad es algo más } \\
\text { que un número }\end{array}$ & $\begin{array}{l}\text { Revisión } \\
\text { sistemática }\end{array}$ & $\begin{array}{c}\text { Trabajadores } \\
\text { del sector de } \\
\text { transporte del } \\
\text { Censo americano, } \\
\text { año 2000 } \\
\text { (4millones) }\end{array}$ & $\begin{array}{l}\text { Tiempo de } \\
\text { respuesta }\end{array}$ \\
\hline Welford A. & 1998 & $\begin{array}{l}\text { La prevención de los } \\
\text { cambios adversos de } \\
\text { trabajo con la edad }\end{array}$ & $\begin{array}{l}\text { Revisión } \\
\text { sistemática }\end{array}$ & & $\begin{array}{c}\text { Nuevos } \\
\text { aprendizajes }\end{array}$ \\
\hline Moyers PA. & 2004 & $\begin{array}{l}\text { Adaptación de los } \\
\text { trabajadores mayores } \\
\text { a los cambios } \\
\text { ocupacionales }\end{array}$ & $\begin{array}{l}\text { Revisión } \\
\text { sistemática }\end{array}$ & & $\begin{array}{l}\text { Criterios de } \\
\text { valoración }\end{array}$ \\
\hline
\end{tabular}

Se debe valorar el área cognitiva usando: Test de Pfeiffer (MEC de Lobo) como complemento y si el individuo es analfabeta el Set Test de Isaacs), así como el Test del informador (TIN corto). Y para la parte afectiva la escala de Depresión de Yesavage?.

Se debe explorar campos de memoria, atención, inteligencia, lenguaje, toma de decisiones, aprendizaje y procesamiento de la información. Debido a la afectación para aprender y realizar múltiples tareas, se deben usar técnicas adecuadas para los nuevos aprendizajes de este grupo etario ${ }^{16,17}$.

En general, la medición de la capacidad mental debe incluir: la capacidad de entender y realizar un trabajo, la capacidad para seguir instrucciones y la capacidad de comunicarse e interactuar. El estado mental de un trabajador no debe comprometer la seguridad de él ni la de otros trabajadores en el lugar de trabajo. Escalas para el examen mental están disponibles y son una herramienta útil en el screening de evaluación del estado mental de los trabajadores, aunque no existen estudios que comparen las diferentes escalas ${ }^{16}$

Partiendo que el trabajador mayor es un individuo físico y mentalmente sano, se debe sin embargo tomar en cuenta dificultades de aprendizaje ligadas no a déficit funcional sino técnicas de aprendizaje inapropiadas o modernas ${ }^{20}$

El deterioro de la memoria puede hacer difícil el aprendizaje de nuevos conceptos, como aquellos asociados a tareas en ordenador o procedimientos operacionales poco comunes. Por otra parte, el deterioro de la capacidad de atención puede dificultar a una persona mayor a realizar tareas comunes o a cambiar su atención entre múltiples mensajes informativos. Encontró que con el aumento de complejidad de una tarea, los adultos mayores son proporcionalmente más lentos que los adultos jóvenes ${ }^{17}$. 
Popkin ${ }^{18}$ reporta que los conductores de camiones menores de 50 años tuvieron significativamente un tiempo de respuesta más rápida $(16$ - 42\%) a través de una variedad de tareas, en comparación con los conductores mayores de 65 años. Además, en su estudio también encuentra que los conductores mayores tienen significativamente un peor desempeño que los conductores jóvenes en la realización de tareas selectivas. El deterioro de la cognición tiene implicaciones para la realización del trabajo, asociado con un deterioro en la velocidad de procesamiento de la información, la cual se acompaña a medida que se avanza en edad. Además, se puede afectar la realización de ciertos trabajos como el deterioro en el campo de la memoria, capacidad de atención y capacidad de percepción. La presión mental a la que se exponen personal sanitario (sirenas, urgencias, trabajo bajo presión del tiempo, toma de decisiones complejas...) no se recomienda para trabajadores mayores ${ }^{6}$. La capacidad de trabajo del trabajador mayor está supeditada a: características personal del trabajador, tareas del trabajo y los retos profesionales ${ }^{21}$.

Tabla 4. Valoración de la capacidad social.

\begin{tabular}{lclcc}
\hline \multicolumn{1}{c}{$\mathbf{1}^{\mathbf{0}}$ Autor } & Año & \multicolumn{1}{c}{ Artículo } & Tipo de estudio & Capacidad física \\
\hline Chang G & 2000 & $\begin{array}{l}\text { Envejecimiento y aptitud para } \\
\text { trabajar }\end{array}$ & $\begin{array}{c}\text { Revisión } \\
\text { sistemática }\end{array}$ & $\begin{array}{c}\text { Debe explorarse } \\
\text { el entorno físico } \\
\text { del trabajador }\end{array}$ \\
\cline { 3 - 5 } & & & & Valoración integral \\
\hline $\begin{array}{l}\text { Servicio } \\
\text { Canario } \\
\text { de Salud }\end{array}$ & 2002 & $\begin{array}{l}\text { Guía de Atención en las personas } \\
\text { Mayores en Atención Primaria }\end{array}$ & Texto completo & Valoración Integral \\
\hline
\end{tabular}

Se debe valorar la relación entre el individuo y su entorno socio-familiar que lo rodea?:

- Núcleo familiar

- Características y estado de la vivienda

- Relaciones y actividades sociales, descartando aislamiento

- Apoyo social y situación económica (ayudas económicas...)

Chang ${ }^{16}$ agrega además en esta fase la búsqueda de síntomas depresivos y señala que la evaluación social es una parte integral de la evaluación funcional en general del adulto mayor.

Tabla 5. Índice de capacidad de trabajo.

\begin{tabular}{cclcc}
\hline \multicolumn{1}{c}{$\mathbf{1}^{\circ}$ Autor } & Año & \multicolumn{1}{c}{ Artículo } & Tipo de estudio & Capacidad física \\
\hline Ilmarinem J. & 2000 & $\begin{array}{l}\text { Envejecimiento de la población } \\
\text { trabajadora de la UE }\end{array}$ & $\begin{array}{c}\text { Revisión } \\
\text { sistemática }\end{array}$ & \\
\hline Chang G & 2000 & $\begin{array}{l}\text { Envejecimiento y aptitud para } \\
\text { trabajar }\end{array}$ & $\begin{array}{c}\text { Revisión } \\
\text { sistemática }\end{array}$ & Uso de WAI \\
\hline Williams S. & 1997 & $\begin{array}{l}\text { La investigación de la capacidad } \\
\text { laboral de los trabajadores mayores }\end{array}$ & $\begin{array}{c}\text { Revisión } \\
\text { sistemática }\end{array}$ & \\
\hline
\end{tabular}

Es un instrumento desarrollado por el Instituto Finlandés de Medicina del Trabajo en 1994, conocido como Work Ability Index (WAI), Índice de capacidad de trabajo. Este índice ayuda a proporcionar una referencia percibida de la capacidad de trabajo de los propios trabajadores mayores en comparación con su mejor tiempo de vida y su capacidad de trabajo en relación con demandas de trabajo. ${ }^{11,16,20}$

Las respuestas a los cuestionarios dan un score total con un rango entre $7-49$ puntos.

- Scores entre 7 - 27: capacidad de trabajo pobres y sugieren que las medidas para restaurar la capacidad de trabajo sean consideradas.

- Scores entre 28 - 43: capacidad de trabajo moderados e implican que las medidas deberían ser tomadas para mejorar la capacidad laboral. 
- Scores entre 44 - 49: capacidad de trabajo buenos y sugieren que las medidas de apoyo sean apuntadas al mantenimiento de la capacidad de trabajo.

El grupo participante consistió en 20 empleados ( 9 hombres y 11 mujeres) entre los 50 a 55 años. Los participantes en este estudio de investigación fueron seleccionados sobre la base de la edad, el género, y la ocupación. Estos criterios de selección fueron usados para incluir participante quienes podrían ser comparados a los trabajadores finlandeses municipales en similares tareas de trabajo. La mayor parte de los participantes en este estudio trabajaron como oficinistas, administradores, instructores, y técnicos ${ }^{20}$. Entrevistas individuales fueron realizadas para recolectar los datos para este estudio. Después recibían una explicación del propósito del estudio, leían una declaración explicativa y firmaban un consentimiento. Cada participante realizaba un cuestionario que contenía preguntas acerca de capacidades físicas, mentales y sociales. El tiempo de promedio para completar el cuestionario fue de 5 a 10 minutos. Se realizan estimaciones subjetivas de la capacidad laboral en relación a enfermedades diagnosticadas, demandas laborares y recursos psicológicos ${ }^{20}$.

El índice también contiene preguntas sobre el ausentismo laboral y enfermedades. Las conclusiones de este estudio de investigación sugieren que el índice de capacidad de trabajo desarrollado por el Instituto finlandés de Salud Profesional sea un instrumento viable para acercarse sobre la capacidad de trabajo de empleados mayores. También, los valores de índice de capacidad de trabajo total pueden ser usados para determinar si hay necesidad de cambios en las tareas de trabajo o exigencias de trabajo ${ }^{20}$.

El análisis comparativo sobre capacidad de trabajo total de la población de la universidad y los valores de referencia desarrollados por el Instituto finlandés de Salud Ocupacional reveló que las personas de género similar, profesión, y la edad en EU tienen valores de capacidad de trabajo ligeramente más altos que los operadores finlandeses municipales y otras profesiones. La comparación de los valores de capacidad de trabajo de empleados de universidad 51-54 con los valores de referencia finlandeses para personas de 50 - 55 años también muestra que los valores de este estudio eran más altos. También las conclusiones formadas usando las posiciones de capacidad de trabajo se diferenciaron. Trabajadores finlandeses municipales concluyeron que su capacidad de trabajo se cayó a la categoría moderada. Sin embargo, usando los valores de capacidad de trabajo calculados, el $85 \%$ de los participantes en esta población tenía una posición de capacidad de trabajo buena $^{20}$.

Aunque las posiciones de capacidad de trabajo de los trabajadores finlandeses se diferenciaran de aquellos obtenidos de los participantes en este estudio, las posiciones obtenidas de esta población no eran considerablemente diferentes. La gama de los valores de capacidad de trabajo calculados para los participantes usados en este estudio de investigación, envejezca 50 a 55 años, era 41-49 puntos. La gama de los valores de capacidad de trabajo calculados para cada uno de los grupos principales profesionales era 44-49 puntos para profesores, 46-47 puntos para técnicos, y 41-48 puntos para oficinistas. Aunque los valores de referencia catalogados en el índice de capacidad de trabajo para empleados finlandeses en profesiones similares diferenciadas de la capacidad de trabajo de los participantes en este estudio, los resultados obtenidos todavía puedan ser usados hacer la determinación sobre la capacidad de trabajo de más viejos empleados de universidad. Los valores de capacidad de trabajo de los participantes en este estudio pueden ser usados identificar medidas preventivas para mantener la capacidad de trabajo. Muchos cambios relacionados con la edad pueden ser sujetos a la modificación, a menudo por la puesta en práctica de estrategias específicas como la buena alimentación, modos de vivir sanos, y entrenarse". Para hacer una determinación válida de capacidad de trabajo, las medidas de continuación deberían ser tomadas con otros procedimientos de selección $^{20}$.

Animan a evaluaciones de continuación periódicas en particular para aquellos participantes diagnosticados con enfermedades cardiovasculares y aquellos participantes con niveles de capacidad de trabajo moderados ${ }^{20}$. 


\section{CONCLUSIONES}

- Existe una tendencia mundial creciente de prolongar la edad de jubilación, que va ligado a un aumento de la esperanza de vida de la población con una disminución de la tasa de natalidad.

- Hoy en día con la necesidad de retrasar la edad de jubilación es imprescindible que el médico de trabajo conozca los riesgos físicos (ergonómicos), psíquicos y sociales a los que se encuentra el trabajador mayor para poder relacionarlo con su entorno laboral (demandas, exigencias y condiciones) y realizar una valoración funcional integral adecuada que permita reconocer si se encuentra en capacidad de continuar sus tareas y/o recomendaciones para evitar mayor deterioro o adaptaciones al puesto de trabajo .

- En trabajadores mayores: hay menor número de accidentes, aunque suelen ser más graves. Presentan menor número de incapacidades temporales, aunque la duración de ellas es mayor debido a la presencia de enfermedades crónicas.

- La mayoría de los artículos encontrados están relacionadas específicamente con patologías crónicas o comorbilidad de los trabajadores envejecidos.

- Muchos artículos informan cambios degenerativos de la edad y las recomendaciones ergonómicas para disminuir su deterioro.

- Nos impresiona que el número de publicaciones relacionadas con la valoración del trabajador mayor es insuficiente.

- No hay estudios publicados específicos de valoración visual, auditivas ni cognitiva en trabajadores mayores.

\section{REFERENCIAS BIBLIOGRÁFICAS}

1. De Zwart BC, Frins-Dresen MH, van Dijk FJ. Physical workload and the aging worker: review of the literature. Int Arch Occup Environ Health.1995;69(1):1-12.

2. Pérez Bilbao J, Nogareda Cuixart C, Salvador Paracaula E. Ministerio de trabajo y asuntos sociales España. Instituto nacional de seguridad e higiene en el trabajo. Envejecimiento y trabajo: La visión. Disponible en: http: // www.insht.es.

3. Winn FJ, Ph.D. An international perspective on the older worker. International Journal of industrial Ergonomics. 2000;20: 461-463.

4. Pérez Bilbao J, Nogareda Cuixart C. Ministerio de trabajo y asuntos sociales España. Instituto nacional de seguridad e higiene en el trabajo. Envejecimiento y trabajo: la gestión de la edad. Disponible en: http: // www.insht.es.

5. Persad CC, CookS, Giordani B. Assessing falls in the elderly: should we use simple screening test or a comprehensive fall risk evaluation?. Eur J Phys Rehabilitation Medicine. 2010;46(2):249-59.

6. Sluiter JK. High-demand jobs: age-related diversity in work ability?. Apply Ergonomy. 2006;37(4):429-40.

7. Shephard RJ. Age and physical work capacity. Exp aging Res.1999;25(4):331-43.

8. Tortosa L, García Molina C, Page A, Ferreras A, Castelló P, Piedrabuena A. Trabajo y envejecimiento Mejora de las condiciones ergonómicas de la actividad laboral para la promoción de un envejecimiento saludable. Instituto de Biomédica de Valencia.2004.

9. Gobierno de Canarias. Guía de actuación en las personas mayores en atención primaria. 2002. www. gobiernocanarias.es.

10. Shephard RJ. Human rights and the older worker: changes in work capacity with age. Med Sci Sports Excerc. 1987;19(2):168-73.

11. Ilmarinen J, Costa G. Aging of the working population in the European Union. Med lav. 2009;91(4):279-95.

12. Welford AT. Preventing adverse changes of work with age. Int J Aging Hum Dev. 1998;27(4):283-91.

13. Lillo Jover J, Moreira Villegas H. Ergonomía y trabajadores mayores. Cuadernos de relaciones laborales.2009;27(2):51-62.

14. Kenny GP, Yardley JE, Martieneau L, Jay O. Physical work capacity in older adults: implications for the aging worker. American journal ind Med. 2008;51(8):610-25. 
15. Parker M, Bergmark E, Philip H. Dell. Functional assessment and the older worker. Top geriatr rehabil. 1994;10(1):39-49.

16. Chan G, Tani V, Koh D. Ageing and fitness to work. Occupational Medicine. 2000;50(7):483-491.

17. Kowalski-Trackofler KM, Steiner LJ, Schwerha DJ. Safety considerations of the aging workforce. Safety Science. 2005; $43: 779-793$

18. Popkin SM, Morrow SL, Di Domenico TE, Howarth HD. Age is more than just a number: Implications for aging workforce in US transportation sector. Applied Ergonomics. 2008; 39:542-549.

19. Davila EP, Caban-Martinez AJ, Muenning P, Lee DJ, Flemin LE, Ferraro KF, et al. Sensory Impairment among older US workers. American journal public health. 2009;99(8):1378-85.

20. Williams SN , Crumpton LL. Investigating the work ability of older employees. International journal of industrial Ergonomic. 1997;20(3):241-249.

21. Moyers PA, Coleman SD. Adaptation of the older worker to occupational challenges. Work. 2004;22(2):71-8.

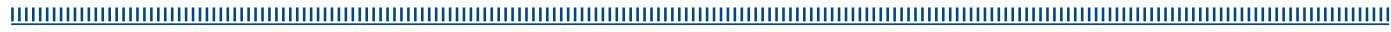

